\title{
Assessing severe asthma
}

\author{
Douglas S. Robinson \\ Affiliation: Severe Asthma Service, Respiratory Medicine, University College London Hospitals NHS Trust, \\ London, UK.
}

Correspondence: Douglas S. Robinson, Severe Asthma Service, Respiratory Medicine, University College London Hospitals NHS Trust, 250 Euston Road, London NW1 2PG, UK. E-mail: douglas.robinsonduclh.nhs.uk

○ @ERSpublications

Structured assessment of patients identifies asthma misdiagnosis, and improves asthma control and quality of life http://ow.ly/Rx3p3017DTx

In the current issue of the European Respiratory Journal, vAN DER MEER et al. [1] describe data from a 1 day assessment of difficult to treat asthma at their specialist centre in the Netherlands. They conclude that the structured assessment identified misdiagnosis, and improved asthma control and quality of life.

It is now 13 years since we and others described results from protocolised assessment of patients seen in specialist centres because of lack of control of their asthma with high dose inhaled corticosteroids (ICS) plus additional controller (usually long acting beta agonists; LABA), or those requiring long term oral steroids to control the disease [2,3]. In that time data have confirmed the usefulness of a systematic approach, and that it improves outcomes [4]. What does it add, other than transferring "heart sink" patients to others? There are several benefits to this approach, as follows.

First, confirmation of the diagnosis of asthma as the cause of continued symptoms. As many as $10 \%$ of patients referred with difficult to treat asthma do not in fact have asthma, and that is confirmed again in the current report, where the figure was 9\% [1-4]. The diagnosis of asthma is not always straightforward. Up to $50 \%$ of those with severe asthma may not have demonstrable reversible airflow obstruction at the time of assessment, some have normal spirometry and, in others, low forced expiratory volume in $1 \mathrm{~s}$ may make assessment of airway hyper-responsiveness difficult. Nonetheless, the lack of documented reversible airway narrowing should lead to reappraisal of the clinical features (for example, a history of chronic sputum production and consistent, nonvariable breathlessness is probably not asthma; it is surprisingly common to see patients labelled with asthma when their history is not suggestive of the diagnosis) and further measurement, including airway hyper-responsiveness. As is suggested in recent draft guidelines from the National Institute for Health and Care Excellence in the UK [5], exhaled nitric oxide fraction ( $F \mathrm{eNO}$ ) may be useful for identification of airway inflammation: the presence of which makes asthma more likely. In addition, protocolised assessment will frequently identify additional diagnoses that need to be assessed: these included bronchiectasis (which may be due to allergic bronchopulmonary aspergillosis), fixed airflow obstruction, dysfunctional breathing, upper airway disease and cardiac problems, etc. [1-4].

What is the "standard" investigation set required? Time to go over a careful and unbiased history is perhaps the most important. A full blood work up, including an eosinophil count, biochemistry, assessment of allergic sensitisation, Aspergillus antibodies, anti-neutrophil cytoplasmic antibodies and, if there are frequent infections, immune function (IgG, IgA, IgM and specific IgG to recall antigens such as Streptococcus pneumoniae and tetanus, depending on local guidelines). Chest radiography and high resolution computed tomography scanning are helpful, both to identify other disease (most commonly bronchiectasis) and to confirm changes of severe asthma, such as air trapping, bronchial wall thickening and mucus plugging.

The second element is to address the commonest cause of lack of response to treatment in any chronic disease: nonadherence. The Belfast group have lead the way in moving from an awkward judgmental guess

Received: May 242016 | Accepted: May 252016

Conflict of interest: Disclosures can be found alongside this article at erj.ersjournals.com

Copyright @ERS 2016 
(more often wrong than not), to an objective measure of adherence [6-8]. For oral prednisolone, serum cortisol and prednisolone should be measured [2]. A review of prescription records can be revealing, although this is easier in some places than others [1,6]. Increasingly, use of FeNO suppression tests, under either supervised treatment [7] or using new recording devices fitted to inhalers, can distinguish persistent airway inflammation due to severe treatment-resistant disease from airway inflammation due to lack of adherence to anti-inflammatory treatment. The results of such tests need careful handling. Abrupt confrontation may lead to the patient going elsewhere (often to repeat the cycle); an multidiscipinary approach with skilled and experienced clinical nurse specialists and psychology input is invaluable for this aspect and for managing the frequent psychosocial factors that affect both symptoms and treatment adherence. Of the 40 patients in the report of VAN DER MEER et al. [1], 41\% were considered nonadherent.

Thirdly, and increasingly importantly, systematic assessment is important in identifying and characterising those patients with uncontrolled severe asthma despite high level (British Thoracic Society step 4/5) treatment. This is usually around $50 \%$ of those assessed (51\% in the paper of VAN DER MeER et al. [1]). As has recently been confirmed, these patient run considerable risk of morbidity from oral steroids, either as frequent short bursts or continuous treatment [9]. Current data suggests that not all asthma is the same and characterising the subtype of asthma may be helpful [10]. Identifying the degree of airway inflammation by blood eosinophil counts (or induced sputum in some centres), and FeNO may guide steroid dosing: the use of such biomarkers to guide treatment has been shown to reduce exacerbation rates [11], and is under assessment in the current UK Medical Research Council RASP (Refractory Asthma Stratification Programme) study [12]. This year, anti-IL-5 (mepolizumab) was licenced for severe asthma, joining anti-IgE (omalizumab) in the armamentarium of biologics for this condition, which will likely grow considerably within the next 10 years. These drugs are expensive, and the challenge for the future is to set up networks of centres of excellence with the facilities and, perhaps more important, the experience, to fully characterise each patient so that medicines are personalised and monitored appropriately.

In England and Wales, the National Health Service has identified specialist centres for severe asthma care to provide assessment and monitor treatment for each region. These centres will act as gate keepers for biologics, but must be seen as enablers for those who require this treatment, rather than barriers to access. Most are still in the process of setting up and it will take time to develop the operational networks required to deliver the best care for all.

The report from VAN DER MEer et al. [1] shows how a 1 day assessment in a specialist centre, in concert with a network of informed pulmonary physicians, primary care providers and patients, can make a difference to care. Most of the patients seen returned to the referring physicians for follow-up, yet at 1 year there was measurable benefit in terms of improvements in asthma control questionnaire, quality of life measures and reduction in exacerbation frequency, albeit this was an open descriptive report. The patients seen were referred from respiratory physicians, but this report suggests that an effective networked approach can work and that it is not necessary for ever-increasing numbers of asthmatics to accumulate under the long term care of specialist centres.

The challenge globally is to identify and screen patients optimally so that they can benefit from expert assessment and treatment in concert with local management.

A perhaps unspoken challenge for those managing asthma is that it is not unlikely that the same principles can be applied to all asthma patients (and probably those with other airway diseases). Misdiagnosis, non-diagnosis, under-treatment and over-treatment need to be addressed across the board, and if there really are different subtypes of airway disease differentially responsive to inhaled steroids and other treatments (as seems likely) we need to develop strategies to address this through access to expert and systematic assessment in an integrated manner at an early stage.

\section{References}

1 van der Meer AN, Pasma H, Kempenaar-Okkema W, et al. A 1-day visit in a severe asthma centre: effect on asthma control, quality of life and healthcare use. Eur Respir J 2016; 48: 726-733.

2 Robinson DS, Campbell DA, Durham SR, et al. Systematic assessment of difficult-to-treat asthma. Eur Respir J 2003; 22: 478-483.

3 Heaney LG, Conway E, Kelly C, et al. Predictors of therapy resistant asthma: outcome of a systematic evaluation protocol. Thorax 2003; 58: 561-566.

4 Sweeney J, Brightling CE, Menzies-Gow A, et al. Clinical management and outcome of refractory asthma in the UK from the British Thoracic Society Difficult Asthma Registry. Thorax 2012; 67: 754-756.

5 National Institute for Health and Care Excellence. Asthma: diagnosis and monitoring. www.nice.org.uk/guidance/ indevelopment/gid-cgwave0640 Date last updated: May 9, 2016.

6 Gamble J, Stevenson M, McClean E, et al. The prevalence of nonadherence in difficult asthma. Am J Respir Crit Care Med 2009; 180: 817-822.

7 McNicholl DM, Stevenson M, McGarvey LP, et al. The utility of fractional exhaled nitric oxide suppression in the identification of nonadherence in difficultasthma. Am J Respir Crit Care Med 2012; 186: 1102-1108. 
8 Heaney LG, Robinson DS. Severe asthma treatment: need for characterising patients. Lancet 2005; 365: 974-976. Sweeney J, Patterson CC, Menzies-Gow A, et al. Comorbidity in severe asthma requiring systemic corticosteroid therapy: cross-sectional data from the Optimum Patient Care Research Database and the British Thoracic Difficult Asthma Registry. Thorax 2016; 71: 339-346.

10 Agusti A, Bel E, Thomas M, et al. Treatable traits: toward precision medicine of chronic airway diseases. Eur Respir J 2016; 47: 410-419.

11 Green RH, Brightling CE, McKenna S, et al. Asthma exacerbations and sputum eosinophil counts: a randomised controlled trial. Lancet 2002; 360: 1715-1721.

12 Heaney LG, Djukanovic R, Woodcock A, et al. Research in progress: Medical Research Council United Kingdom Refractory Asthma Stratification Programme (RASP-UK). Thorax 2016; 71: 187-189. 